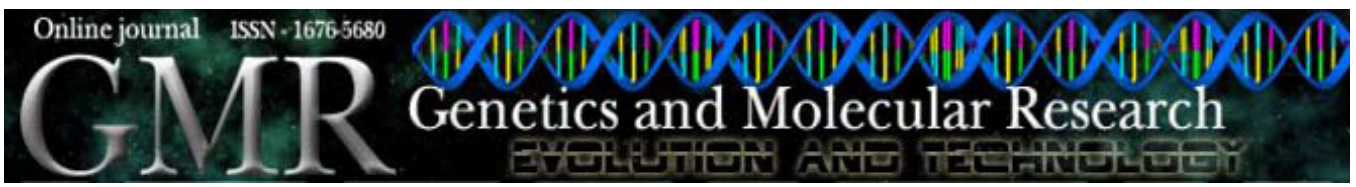

Thesis Abstract

\title{
Comparative cytogenetics study between Triatoma maculata and Triatoma pseudomaculata (Triatominae, Heteroptera)
}

\author{
Weverson Luciano Pires \\ 2008. Instituto de Biociências, Letras e Ciências Exatas, \\ Universidade Estadual Paulista, São José do Rio Preto, SP, Brasil. \\ Master's thesis. Orienting Professor: Dr. Maria Tercília Vilela de Azeredo-Oliveira
}

Triatomines are vectors of the protozoan Trypanosoma cruzi, the etiological agent of Chagas' disease. These hematophagous insects belong to the order Heteroptera and to the family Reduviidae. Disseminated throughout large portions of Brazil and of other Latin American countries, Chagas' disease is a serious and important public health problem that is difficult to treat. Cytogenetically, the interest in triatomines is in its holocentric chromosomes and in its uncommon meiosis process; sexual segregation is post-reductional. The basic number of chromosomes in triatomines is $2 \mathrm{n}=22$. We analyzed spermatogenesis in Triatoma maculata and T. pseudomaculata, with emphasis on the following aspects: spermatogenesis phases, chromatin and meiotic chromosome structure and the nucleolar cycle. These species are found principally in northeastern Brazil and are potential vectors for T. cruzi. The specimens were supplied by the insectary of the Special Health Service of Araraquara (SESA), Department of Epidemiology, School of Public Health, University of São Paulo. Cells were obtained from the testicles of adult males; after crushing they were submitted to the following cytochemical techniques: lacto-acetic orcein staining, impregnation with silver ions, C-banding and critical electrolyte concentrations. An association was observed between the nucleolus and heterochromatic regions, in both interphasic somatic cells and germ line. The localization of these specific regions also demonstrated an intimate functional relationship between these structures. The karyograms of the two species, mounted at metaphase I, indicated 10 autosomes and one pair of sexual chromosomes (22 chromosomes), coinciding with the modal number for the group. With C-banding, the Y chromosome is heterochromatic in both species. During the meiotic cycle, nucleolar activity persists until the end of prophase I (diplotene-diakinesis), when this structure fragments into small corpuscles (particles of ribonucleoproteins). From the initial phases of spermatogenesis until the spermatid elongation phase, one ribonucleoprotein corpuscle is visible that is analogous to the chromatoid body found in vertebrate spermatogenesis. 